\title{
Dose-Dense Regimen of Cisplatin and Infusional Fluorouracil in Advanced Gastric Cancer-A Single Institution Experience
}

\author{
Ofer Purim $^{1 * \#}$, Yulia Kundel ${ }^{1 *}$, Roni Shapira ${ }^{2}$, Thomas Tichler $^{3}$, Baruch Brenner $^{1}$ \\ ${ }^{1}$ Institute of Oncology, Davidoff Center, Rabin Medical Center, Petach Tiqwa, and Sackler Faculty of Medicine, Tel Aviv University, \\ Tel Aviv, Israel; ${ }^{2}$ Institute of Oncology, Chaim Sheba Medical Center, Tel Hashomer, Israel; ${ }^{3}$ Institute of Oncology, Shaare Zedek \\ Medical Center, Jerusalem, Israel. \\ Email:" ozike@walla.co.il
}

Received November $24^{\text {th }}, 2012$; revised December $26^{\text {th }}, 2012$; accepted January $2^{\text {nd }}, 2013$

Copyright (C) 2013 Ofer Purim et al. This is an open access article distributed under the Creative Commons Attribution License, which permits unrestricted use, distribution, and reproduction in any medium, provided the original work is properly cited.

\begin{abstract}
Chemotherapy with continuous infusion of 5-fluorouracil and cisplatin in a monthly schedule is one of the most common regimens in the treatment of advanced gastric cancer. In this study, we evaluated the efficacy and safety of a dosedense administration of this regimen in this patient population. Sixty-six consecutive patients with previously untreated histologically confirmed unresectable or metastatic gastric adenocarcinoma were treated with a 2-hour infusion of cisplatin $100 \mathrm{mg} / \mathrm{m}^{2}$ followed by continuous infusion of 5 -fluorouracil $1000 \mathrm{mg} / \mathrm{m}^{2} /$ day for 5 days, every 21 days. The most common grade $\geq 3$ toxicities were fatigue $(42 \%)$, nausea/vomiting $(30 \%)$ and leucopenia $(12 \%)$. Four patients $(6 \%)$ died from treatment-related toxicity. The response rate was $35 \%$, the median progression-free survival was 4.3 months and the median survival was 5.9 months. In light of these results, the dose-dense approach seems to offer little, if any, benefit compared with the standard regimens.
\end{abstract}

Keywords: Gastric Cancer; Dose-Dense; Cisplatin; Fluorouracil

\section{Introduction}

Adenocarcinoma of the stomach is one of the leading causes of cancer death worldwide. Complete resection is the only curative treatment but approximately two-thirds of patients present with unresectable primary tumor or overt metastatic spread [1]. Furthermore, recurrent disease is noted in up to half of the patients undergoing curative surgery [2-4]. Patients with metastatic disease have a dismal prognosis with a median survival of 5 to 10 months [5-9]. In spite of the fact that multiple chemotherapeutic agents have been studied in advanced gastric cancer (AGC) since the 1970's, either alone or in combination, the median survival in AGC has not changed significantly.

A very common regimen in AGC used today is a combination of continuous infusion (CI) 5-fluorouracil (5FU) and cisplatin, known as FUP. 5FU and cisplatin have been shown to possess synergistic cytotoxic effects against various human tumors including gastric cancer $[10,11]$.

\footnotetext{
*O. P. and Y. K. contributed equally to this manuscript.

\#Corresponding author.
}

Cisplatin is assumed to enhance the antitumor effect of $5 \mathrm{FU}$ by increasing the availability of the reduced folate necessary for tight binding of fluorodeoxyuridylate, a 5FU metabolite, to deoxythymidylic acid synthase [12, 13]. In addition, several studies and a meta-analysis have shown that $5 \mathrm{FU}$ is more effective when administered by CI rather than by bolus injection in several types of cancer including AGC [14-17]. Based on these facts, FUP was evaluated in AGC with good results, and has become one of the most frequently used regimens in this disease [18, 19].

One of the strategies to improve the efficacy of chemotherapeutic regimens is to increase their dose density, i.e., to decrease the interval between treatments. This approach is based on the hypothesis that maximal effectiveness can be achieved by scheduling the chemotherapy intervals to correspond with the period of most rapid tumor growth. Cumulative data, mainly from breast cancer and lymphomas, suggest that dose density can lead to an improved patient outcome without a significant increase in toxicity [20-23]. 
The dose-dense approach was also evaluated in AGC. In a study by Cascinu et al. [24], 105 patients with AGC were treated with a dose-dense regimen of $5 \mathrm{FU}$, cisplatin, epi-doxorubicin, 6S-leucovorin and glutathione, with the support of stem cell growth factors. The results were encouraging: overall response rate was $62 \%$, the median survival duration was 11 months and the 1- and 2-year survival rates were $42 \%$ and $5 \%$, respectively [24].

In the light of the lack of a clear standard regimen in AGC and the favorable results of the dose-dense strategy in other tumors, we decided to adopt this approach in this disease. A dose-dense FUP regimen, given every three instead of four weeks, has already been used in localized and in advanced head and neck cancer $[25,26]$ with a reasonable toxicity profile and we therefore chose this regimen as our standard in AGC. We hereby present our experience using this approach and include an analysis of factors predicting toxicity and benefit from treatment.

\section{Patients and Methods}

\subsection{Eligibility}

All patients had histologically confirmed inoperable or metastatic adenocarcinoma of the stomach or gastroesophageal junction (GEJ). Other inclusion criteria were an Eastern Cooperative Oncology Group performance status (PS) $\leq 3$; measurable or evaluable disease; no prior chemotherapy; adequate bone marrow function (defined as leukocyte count $>4000$ cells $/ \mathrm{mm}^{3}$ and platelet count $>100,000$ cells $/ \mathrm{mm}^{3}$ ); normal liver function (defined as a total bilirubin $<1.5 \mathrm{mg} / \mathrm{dL}$, serum aspartate transaminase and alkaline phosphatase $<3$ times the upper normal limit); and normal renal function (defined as creatinine clearance $>60 \mathrm{cc} / \mathrm{min}$ ). Informed consent was obtained from all patients prior to start of treatment.

Exclusion criteria included a PS of 4, prior chemotherapy, severe co-morbidities, other primary tumors (aside of basal cell carcinoma of the skin and carcinoma in situ of cervix uteri), and inability to sign an informed consent.

\subsection{Patient Evaluation}

Patient evaluation prior to the initiation of treatment included complete medical history and physical examination, complete blood count (CBC), serum chemistry and creatinine clearance test (CCT), serum tumor markers (carcinoembryonic antigen [CEA] and CA-19.9), and chest and abdominal computerized tomography (CT). During treatment, patients were evaluated for toxicity before each cycle, including full medical history and physical examination in addition to $\mathrm{CBC}$, serum chemistry and CCT. Evaluation of efficacy, including the per- formance of tumor markers and CT, was done every 2 - 3 courses according to the judgment of the treating physician.

\subsection{Treatment}

Patients were treated with cisplatin $100 \mathrm{mg} / \mathrm{m}^{2}$ in a 2 hour infusion with adequate prehydration on day 1 followed by CI $5 \mathrm{FU} 1000 \mathrm{mg} / \mathrm{m}^{2} /$ day on days $1-5$. Antiemetic therapy consisted of 5-hydroxytryptamine-3 receptor antagonists and dexamethasone. The regimen was repeated every 21 days.

\subsection{Assessment of Response and Toxicity}

Responses were classified according to World Health Organization (WHO) criteria. A complete response (CR) was defined as the disappearance of all measurable lesions with no new lesions for at least 4 weeks. A partial response (PR) was defined as a reduction of at least $50 \%$ in the sum of the products of the longest perpendicular diameters of all measurable lesions and the absence of new lesions for at least 4 weeks. Stable disease (SD) was defined as a reduction of less than $50 \%$ or an increase of less than $25 \%$ in the sum of the products of the perpendicular diameters of all lesions without any evidence of new lesions for at least 4 weeks. Progressive disease (PD) was defined as an increase of greater than $25 \%$ in tumor size or the appearance of new lesions. Toxicities were graded according to the CTC-NCI version 2.

\subsection{Statistics}

Overall survival (OS) was calculated from the start of treatment to death or the last date the patient was known to be alive. Progression-free survival (PFS) was calculated from the start of treatment to the date of documented progression or the last date the patient was known to be progression-free. The product limit estimated method (Kaplan-Meier) was used to estimate OS and PFS intervals. In order to compare response to treatment, OS and PFS according to various clinicopathological factors, Chi-Square and log-rank test were used. A pvalue of less or equal to 0.05 were considered statistically significant.

\section{Results}

Sixty-six patients were enrolled in the study. Patient characteristics are summarized in Table 1. The median age was 65 years (range: 33 - 93) and the majority of patients were male $(67 \%)$. Sixty-five percent of the patients had a good PS $(0-1)$. The majority of patients $(89 \%)$ had metastatic disease, which almost invariably involved the liver, omentum or both. 
Table 1. Patient characteristics $(n=66)$.

\begin{tabular}{|c|c|c|}
\hline & No. & $\%$ \\
\hline \multicolumn{3}{|l|}{ Age, years } \\
\hline Median & 65 & \\
\hline Range & $33-93$ & \\
\hline \multicolumn{3}{|l|}{ Gender } \\
\hline Male & 44 & 67 \\
\hline Female & 22 & 33 \\
\hline \multicolumn{3}{|l|}{ Performance status } \\
\hline 0 & 10 & 15 \\
\hline 1 & 33 & 50 \\
\hline 2 & 13 & 20 \\
\hline 3 & 10 & 15 \\
\hline \multicolumn{3}{|l|}{ Extent of disease } \\
\hline Locally advanced & 7 & 11 \\
\hline Metastatic & 59 & 89 \\
\hline \multicolumn{3}{|l|}{ Grade } \\
\hline I & 1 & 2 \\
\hline II & 8 & 12 \\
\hline III & 33 & 50 \\
\hline IV & 11 & 17 \\
\hline Unknown & 13 & 19 \\
\hline \multicolumn{3}{|l|}{ Sites of disease } \\
\hline Liver & 35 & 53 \\
\hline Lymph nodes & 31 & 47 \\
\hline Omentum & 27 & 41 \\
\hline Local recurrence & 16 & 24 \\
\hline Omentum and liver & 9 & 14 \\
\hline
\end{tabular}

\subsection{Safety and Dose Intensity}

All 66 patients received at least one cycle of chemotherapy and were assessable for toxicity and dose-intensity (DI) analysis. The median number of treatment cycles administered was 3 (range, 1 - 6). The median DI of 5FU and cisplatin was $90 \%$ (range, $49 \%-100 \%$ ) and $93 \%$ (range, 58\% - 100\%) of the planned dose, respectively.

The toxicities observed in the study population are listed in Table 2. Chemotherapy was associated with significant toxicity. Treatment was discontinued due to toxicity in 12 patients $(18 \%)$ and due to patient request in 2 others $(3 \%)$. There were 4 toxic deaths (6\%): 2 patients died from neutropenic sepsis and 2 from diarrhea. The most common overall toxicities were fatigue, leucopenia and mucositis, while the most common grade $\geq 3$ toxicities were fatigue, nausea/vomiting and leucopenia, noted in $42 \%, 30 \%$ and $12 \%$ of the patients, respectively.

The two variables which were found to predict toxicity were PS at the start of treatment and age. Poor PS $(\geq 2)$ was associated with an increased risk for grade $\geq 3$ hematological toxicity including leucopenia $(p=0.03)$, anemia $(p=0.003)$ and thrombocytopenia $(p=0.013)$, as well as for severe fatigue ( $p=0.028)$. Age $\geq 65$ was associated with increased grade $\geq 3$ vomiting $(p=0.04)$, anemia $(p=0.05)$ and leucopenia $(p=0.05)$.

\subsection{Response to Treatment}

Forty-eight of the 66 patients $(73 \%)$ were evaluable for response. Sixteen patients (24\%) were unevaluable due to early stoppage of treatment because of toxicity or rapid clinical deterioration and $2(3 \%)$ were lost to follow-up before evaluation. Tumor responses to chemotherapy are summarized in Table 3. Response was noted in 17 of the 48 patients, including one CR (2\%), with an overall objective response rate of $35 \%$. Eleven patients $(23 \%)$ had SD for at least one month as their best response. The rate of disease control $(\mathrm{CR}+\mathrm{PR}+\mathrm{SD})$ was therefore $58 \%$.

We examined the relationship between various clinicopathological factors, including PS, stage, grade, age and gender, and the response to FUP therapy. On univariate analysis, response to treatment was found to correlate with PS $(p=0.0001)$ and the presence of liver metastases $(p=0.002)$. However, in multivariate analysis only the presence or absence of liver metastases retained statistical significance $(p=0.006)$. Liver metastases showed a higher response rate $(65 \%)$ than omental $(38 \%)$ or lymph node metastases (45\%).

Table 2. Incidence of toxicity.

\begin{tabular}{ccc}
\hline Type of toxicity & \multicolumn{2}{c}{ Number of patients (\%) } \\
\hline Hematological & All grades & Grade $>3$ \\
Leucopenia & $49(74)$ & $15(12)$ \\
Neutropenia & $10(15)$ & $2(3)$ \\
Neutropenic fever & $2(3)$ & $2(3)$ \\
Thrombocytopenia & $20(30)$ & $3(4)$ \\
Anemia & $20(30)$ & $7(11)$ \\
Non-hematological & & \\
Mucositis & $29(44)$ & $7(11)$ \\
Diarrhea & $19(29)$ & $5(8)$ \\
Renal & 0 & 0 \\
Peripheral neuropathy & $10(15)$ & $1(2)$ \\
Hand foot syndrome & $1(2)$ & $1(2)$ \\
Hearing loss & $9(14)$ & $1(2)$ \\
Fatigue & $62(94)$ & $28(42)$ \\
Nausea/vomiting & $54(82)$ & $20(30)$ \\
\hline
\end{tabular}


Table 3. Overall objective response rate.

\begin{tabular}{cc}
\hline Response & No (\%) \\
\hline CR + PR & $17(35)$ \\
CR & $1(2)$ \\
PR & $16(33)$ \\
SD & $11(23)$ \\
PD & $20(42)$ \\
\hline
\end{tabular}

\subsection{Survival}

The median OS of the entire group was 5.9 months (range 1 - 29) and the median PFS was 4.3 months (range 0 - 26). On univariate and multivariate analyses, factors that correlated with poor survival were presence of liver metastases (HR $=1.7, \mathrm{p}=0.0546)$ and poor PS $(\mathrm{HR}=$ $2.4, \mathrm{p}=0.0001)$. PS was also a prognostic factor for PFS $(\mathrm{HR}=1.88, \mathrm{p}=0.0001)$.

\section{Discussion}

The current study summarizes the results of an attempt that we have made to adopt the dose-density concept into the treatment of AGC. While the data presented were not produced within a framework of a prospective study, they still provide the only piece of information available to date on the potential impact of this approach on the performance of a very common regimen, FUP, in AGC. In the only prior use of a dose-dense regimen in AGC, Cascinu et al. evaluated an investigational combination of 5FU, cisplatin, epidoxorubicin, 6S-leucovorin and glutathione [24].

So, does a dose-dense FUP regimen, as used in our study, represent a potentially improved FUP regimen? It appears that the answer is a resounding no; response rate of $35 \%$, median PFS of 4.3 months and median OS of 5.9 months, as noted in our study, are at best equal to those obtained by standard FUP regimens in AGC. For example, in two phase II trials, standard FUP yielded response rates of $41 \%$ and $43 \%$ and a median survival of 9 months $[18,19]$. Data from phase III randomized trials is in line with the phase II data. In a study from Korea, where FUP was compared with $5 \mathrm{FU}$ alone or a combination of $5 \mathrm{FU}$, doxorubicin and mitomycin $\mathrm{C}$ (FAM), the response rate in the FUP arm was $51 \%$ and the median survival was 9 months [27].

In another phase III trial, by the European Organization for Research and Treatment of Cancer (EORTC), where FUP was compared with combinations of epirubicin, 5FU and leucovorin (ELF) or 5FU, doxorubicin and methotrexate (FAMTX), the response rate of FUP was $20 \%$ and the median survival was 7.2 months [28]. Similar results were noted in the control arm of the V325 trial, evaluating the addition of Docetaxel to FUP [29].

The toxicity profile of the dose-dense FUP also did not seem to compare favorably with standard FUP regimens. In fact, the incidence and severity of toxic events in the dose-dense FUP were higher than those observed with standard FUP $[18,19]$. Most notably, the rate of treatment-related deaths recorded in our study $(6 \%)$ is unreasonably high.

The lack of enhanced efficacy and the higher toxicity of the dose-dense FUP regimen may be related to two main possible causes: an innate inferiority of the modified regimen or substantial differences between our patient population and those treated in the studies using the standard regimen. Our study population could indeed be considered a poor-risk group, especially when compared with the patients treated in the non-randomized phase II trials: $52 \%$ of our patients were 65 years or older, $15 \%$ had PS $=3,67 \%$ had poorly differentiated tumors, and $53 \%$ had liver metastases. It is unclear, however, if the disappointing results of the dose-dense FUP regimen merely represent the difference between an unselected patient population treated in daily practice to those being treated within clinical trials, or an inferior treatment approach, or both. To put our results in perspective, a comparison of the main patient characteristics and toxicities, as well as efficacy end-points, between our study and several studies using standard FUP regimens, are summarized in Table 4.

In this study we also evaluated prognostic factors predicting response to treatment and survival. Presence of liver metastases was the only factor that correlated with response to treatment. The finding that liver metastases respond better to chemotherapy is in concordance with the results reported by Kondo et al. [30]. However, in two other studies, a lower response rate for liver metastases compared with the primary lesion or lymph nodes was reported [31,32]. Similar to other studies, we also did not find a significant correlation between response rate and PS [33-35].

Prognostic factor analysis for patient outcome identified the presence of liver metastases and poor PS to adversely affect survival. These results are comparable with prior reports [36-38]. Other widely accepted prognostic factors, such as tumor grade, presence of bone metastases, peritoneal metastases and ascites, did not show a prognostic impact in the current study $[39,40]$. Of note is the fact that liver metastases respond better to chemotherapy but are at the same time associated with dismal prognosis, emphasizing the aggressive biology of AGC characterized by significant yet transitory chemosensitivity.

\section{Conclusion}

The dose-dense FUP regimen, as used in our study, failed 
Table 4. Comparison of the current study with studies using standard FUP regimens.

\begin{tabular}{|c|c|c|c|c|}
\hline & $\begin{array}{l}\text { Current } \\
\text { Study }\end{array}$ & $\begin{array}{c}\text { Van Cutsem } \\
\text { et al. [29] }\end{array}$ & $\begin{array}{l}\text { Vanhoefer } \\
\text { et al. [28] }\end{array}$ & $\begin{array}{c}\text { Lacave et } \\
\text { al. }[18]\end{array}$ \\
\hline \multicolumn{5}{|c|}{ Patient/tumor characteristics } \\
\hline \multicolumn{5}{|l|}{ Age (yrs) } \\
\hline Range & $33-93$ & $25-76$ & $24-74$ & $32-74$ \\
\hline Median & 65 & 55 & 57 & 57 \\
\hline$\geq 65$ & $52 \%$ & $25 \%$ & - & - \\
\hline \multicolumn{5}{|l|}{ PS } \\
\hline 2 & $15 \%$ & $1 \%$ & $15 \%$ & $50 \%$ \\
\hline 3 & $15 \%$ & $0 \%$ & $0 \%$ & $0 \%$ \\
\hline PD Pathology & $67 \%$ & - & - & $21 \%$ \\
\hline Metastatic disease & $89 \%$ & $97 \%$ & $80 \%$ & $81 \%$ \\
\hline \multicolumn{5}{|c|}{ Toxicity $($ grade $\geq 3$ ) } \\
\hline Leucopenia & $12 \%$ & $3 \%$ & $17 \%$ & $2 \%$ \\
\hline Neutropenia & $3 \%$ & $57 \%$ & $35 \%$ & $1 \%$ \\
\hline Neutropenic fever & $3 \%$ & $12 \%$ & $5 \%$ & $1 \%$ \\
\hline Fatigue & $42 \%$ & $14 \%$ & - & - \\
\hline Nausea/vomiting & $30 \%$ & $34 \%$ & $26 \%$ & $36 \%$ \\
\hline Renal & 0 & 0 & 2 & 0 \\
\hline Neurotoxicity & $2 \%$ & $3 \%$ & $1 \%$ & $11 \%$ \\
\hline Mucositis & $11 \%$ & $27 \%$ & $12 \%$ & $23 \%$ \\
\hline Diarrhea & $8 \%$ & $8 \%$ & $6 \%$ & - \\
\hline Toxic death & $6 \%$ & $5.2 \%$ & $1.5 \%$ & 0 \\
\hline \multicolumn{5}{|c|}{ Efficacy } \\
\hline Response rate & $35 \%$ & $25 \%$ & $20 \%$ & $41 \%$ \\
\hline Median PFS (months) & 4.3 & 3.7 & 4.1 & 10.2 \\
\hline Median OS (months) & 5.9 & 8.6 & 7.2 & 10.6 \\
\hline
\end{tabular}

to present a potential progress in the treatment of AGC. In the light of the obvious limitations of our retrospective data, it is currently unclear whether the dose-dense strategy has indeed no role in this disease. In any case, we find no reason at the present to replace the standard fourweekly FUP regimen, particularly with the increased quality of life afforded by the longer interval between treatments.

\section{REFERENCES}

[1] J. T. Diehl, R. E. Hermann, A. M. Cooperman and S. O. Hoerr, "Gastric Carcinoma: A Ten-Year Review," Annals of Surgery, Vol. 198, No. 1, 1983, pp. 9-12. doi:10.1097/00000658-198307000-00003

[2] G. McNeer, H. Vandenberg, F. Y. Donn and L. Bowden, "A Critical Evaluation of Subtotal Gastrectomy for the Cure of Cancer of the Stomach," Annals of Surgery, Vol. 134, No. 1, 1951, pp. 1-7.

\section{doi:10.1097/00000658-195107000-00002}

[3] L. L. Gunderson and H. Sosin, "Adenocarcinoma of the Stomach: Areas of Failure in a Reoperation Series (Second or Symptomatic Look) - Clinicopathologic Correlation and Implications for Adjuvant Therapy," International Journal of Radiation Oncology * Biology * Physics, Vol. 8, No. 1, 1982, pp. 1-11. doi:10.1016/0360-3016(82)90377-7

[4] J. B. Dupont Jr., J. R. Lee, G. R. Burton and I. Cohn, Jr., "Adenocarcioma of the Stomach: Review of 1497 Cases," Cancer, Vol. 41, No. 3, 1978, pp. 941-947. doi:10.1002/1097-0142(197803)41:3<941::AID-CNCR28 20410323>3.0.CO;2-M

[5] S. Pyrhönen, T. Kuitunen and M. Kouri, "A Randomized Phase III Trial Comparing Fluorouracil, Epidoxorubicin and Methotrexate (FEMTX) with Best Supportive Care in Non-Resectable Gastric Cancer," Annals of Oncology, Vol. 71, No. 3, 1995, pp. 587-591.

[6] B. Glimelius, K. Ekström, K. Hoffman, W. Graf, P. O. Sjödén, U. Haglund, et al., "Randomized Comparison between Chemotherapy plus Best Supportive Care with Best Supportive Care in Advanced Gastric Cancer," Annals of Oncology, Vol. 8, No. 2, 1997, pp. 163-168. doi:10.1023/A:1008243606668

[7] A. M. Murad, F. F. Santiago, A. Petroianu, P. R. Rocha, M. A. Rodrigues and M. Rausch, "Modified Therapy with 5-Fluorouracil, Doxorubicin and Methotrexate in Advanced Gastric Cancer," Cancer, Vol. 72, No. 1, 1993, pp. $37-41$.

doi:10.1002/1097-0142(19930701)72:1<37::AID-CNCR2 820720109>3.0.CO;2-P

[8] J. A. Akoh and I. M. C. Mcintyre, "Improving Survival in Gastric Cancer: Review of 5-Year Survival Rates in English Language Publications from 1970," British Journal of Surgery, Vol. 79, No. 4, 1992, pp. 293-299. doi:10.1002/bjs. 1800790404

[9] J. Wils, H. Klein, D. Wagener, H. Bleiberg, H. Reis, F. Korsten, et al., "Sequential High-Dose Methotrexate and Fluorouracil Combined with Doxorubicin: A Step Ahead in the Treatment of Advanced Gastric Cancer-A Trial of the EORTC Gastrointestinal Tract Cooperative Group," Journal of Clinical Oncology, Vol. 9, No. 5, 1991, pp. 827 831.

[10] F. M. Schabel, M. W. Trader, W. R. Laster, T. H. Corbett and D. P. Griswold Jr., "Cis-Dichlorodiammineplatinum (II): Combination Chemotherapy and Cross-Resistance Studies with Tumors of Mice," Cancer Treatment Reports, Vol. 63, No. 9-10, 1979, pp. 1459-1473.

[11] M. Rooney, J. Kish, J. Jacobs, J. Kinzie, A. Weaver, J. Crissman, et al., "Improved Complete Response Rate and Survival in Advanced Head and Neck Cancer after Three-Course Induction Therapy with 120-Hour 5-FU Infusion and Cisplatin," Cancer, Vol. 55, No. 5, 1985, pp. 1123-1128. doi:10.1002/1097-0142(19850301)55:5<1123::AID-CNC $\underline{\mathrm{R} 2820550530>3.0 . \mathrm{CO} ; 2-8}$

[12] K. J. Scanlon, E. M. Newman, Y. Lu and D. G. Priest, "Biochemical Basis for Cisplatin and 5-Fluorouracil Syn- 
ergism in Human Ovarian Carcinoma Cells," Proceedings of the National Academy of Sciences of the United States of America, Vol. 83, No. 23, 1986, pp. 8923-8925. doi:10.1073/pnas.83.23.8923

[13] T. Shirasaka, Y. Shimamoto, H. Ohshima, H. Saito and M. Fukushima, "Metabolic Basis of the Synergistic Antitumor Models in Vivo," Cancer Chemotherapy and Pharmacology, Vol. 32, No. 3, 1993, pp. 167-172. doi:10.1007/BF00685830

[14] J. J. Lokich, J. D. Ahlgren, J. J. Gullo, J. A. Phillips and J. G. Fryer, "A Prospective Randomized Comparison of Continuous Infusion Fluorouracil with a Conventional Bolus Schedule in Metastatic Colorectal Carcinoma: A Mid-Atlantic Oncology Program Study," Journal of Clinical Oncology, Vol. 7, No. 4, 1989, pp. 425-432.

[15] T. Moynihan, R. Hansen, T. Anderson, E. Quebbeman, P. Beatty, R. Ausman, et al., "Continuous 5-Fluorouracil Infusion in Advanced Gastric Carcinoma," American Journal of Clinical Oncology, Vol. 11, No. 4, 1988, pp. 461464. doi:10.1097/00000421-198808000-00010

[16] J. A. Kish, J. F. Ensley, J. Jacobs, A. Weaver, G. Cummings and M. Al-Sarraf, "A Randomized Trial of Cisplatin +5 -FU Infusion and Cisplatin +5-FU Bolus for Recurrent and Advanced Squamous Cell Carcinoma of Head and Neck," Cancer, Vol. 56, No. 12, 1985, pp. 27402744.

doi:10.1002/1097-0142(19851215)56:12<2740::AID-CN CR2820561203>3.0.CO;2-Y

[17] R. M. Hansen, L. Ryan, T. Anderson, B. Krzywda, E. Quebbeman, A. Benson 3rd, et al., "Phase III Study of Bolus versus Infusion Fluorouracil with or without Cisplatin in Advanced Colorectal Cancer," Journal of the National Cancer Institute, Vol. 88, No. 10, 1996, pp. 668674. doi:10.1093/jnci/88.10.668

[18] A. J. Lacave, F. J. Barón, L. M. Antón, E. Estrada, L. M. De Sande, I. Palacio, et al., "Combination Chemotherapy with Cisplatin and 5-Fluorouracil 5-Day Infusion in the Therapy of Advanced Gastric Cancer: A Phase II Trial," Annals of Oncology, Vol. 2, No. 10, 1991, pp. 751-754.

[19] P. Rougier, M. Ducreux, M. Mahjoubi, J. P. Pignon, S. Bellefqih, J. Oliveira, et al., "Efficacy of Combined 5-Fluorouracil and Cisplatinum in Advanced Gastric Carcinomas: A Phase II Trial with Prognostic Factor Analysis," European Journal of Cancer, Vol. 30, No. 9, 1994, pp. 1263-1269. doi:10.1016/0959-8049(94)90170-8

[20] S. Kummel, M. Rezai, R. Kimmig and P. Schmid, "DoseDense Chemotherapy for Primary Breast Cancer," Current Opinion in Obstetrics and Gynecology, Vol. 19, No. 1, 2007, pp. 75-81. doi:10.1097/GCO.0b013e328011f99a

[21] L. Anderson-Reitz, "Dose-Dense Chemotherapy for Aggressive Non-Hodgkin Lymphoma," Cancer Nursing, Vol. 29, No. 3, 2006, pp. 198-206. doi:10.1097/00002820-200605000-00005

[22] V. Diehl, J. Franklin, D. Hasenclever, H. Tesch, M. Pfreundschuh, B. Lathan, et al., "BEACOPP, a New Dose-Escalated and Accelerated Regimen, Is at Least as Effective as COPP/ABVD in Patients with Advanced-Stage Hodgkin's Lymphoma: Interim Report from a Trial of the German Hodgkin's Lymphoma Study Group," Journal of
Clinical Oncology, Vol. 16, No. 12, 1998, pp. 3810-3821.

[23] M. L. Citron, D. A. Berry, C. Cirrincione, C. Hudis, E. P. Winer, W. J. Gradishar, et al., "Randomized Trial of DoseDense versus Conventionally Scheduled and Sequential versus Concurrent Combination Chemotherapy as Postoperative Adjuvant Treatment of Node-Positive Primary Breast Cancer: First Report of Intergroup Trial C9741/ Cancer and Leukemia Group B Trial 9741," Journal of Clinical Oncology, Vol. 21, No. 8, 2003, pp. 1431-1439. doi:10.1200/JCO.2003.09.081

[24] S. Cascinu, R. Labianca, P. Alessandroni, M. Marcellini, R. R. Silva, G. Pancera, et al., "Intensive Weekly Chemotherapy for Advanced Gastric Cancer Using Fluorouracil, Cisplatin, Epi-Doxorubicin, 6S-Leucovorin, Glutathione, and Filgrastim: A Report from the Italian Group for the Study of Digestive Tract Cancer," Journal of Clinical Oncology, Vol. 15, No. 11, 1997, pp. 3313-3319.

[25] J. R. Jacobs, K. K. Fu, L. D. Lowry, Scotte R. L. Doggett, T. F. Pajak and M. Al-Sarraf, "5-Year Results of Cisplatin and Fluorouracil Infusion in Head and Neck Cancer," Archives of Otolaryngology-Head and Neck Surgery, Vol. 117, No. 3, 1991, pp. 288-291. doi:10.1001/archotol.1991.01870150056006

[26] M. Al-Sarraf, K. Martz, A. Herskovic, L. Leichman, J. S. Brindle, V. K. Vaitkevicius, et al., "Progress Report of Combined Chemoradiotherapy versus Radiotherapy Alone in Patients with Esophageal Cancer: An Intergroup Study," Journal of Clinical Oncology, Vol. 15, No. 1, 1997, pp. 277-284.

[27] N. K. Kim, Y. S. Park, D. S. Heo, C. Suh, S. Y. Kim, K. C. Park, et al., "A Phase III Randomized Study of 5-Fluorouracil and Cisplatin versus 5-Fluorouracil, Doxorubicin, and Mitomycin $\mathrm{C}$ versus 5-Fluorouracil Alone in the Treatment of Advanced Gastric Cancer," Cancer, Vol. 71, No. 12,1993 , pp. 3813-3818.

doi:10.1002/1097-0142(19930615)71:12<3813::AID-CN CR2820711205>3.0.CO;2-5

[28] U. Vanhoefer, P. Rougier, H. Wilke, M. P. Ducreux, A. J. Lacave and E. Van Cutsem, et al., "Final Results of a Randomized Phase III Trial of Sequential High-Dose Methotrexate, Fluorouracil, and Doxorubicin versus Etoposide, Leucovorin, and Fluorouracil versus Infusional Fluorouracil and Cisplatin in Advanced Gastric Cancer: A Trial of the European Organization for Research and Treatment of Cancer Gastrointestinal Tract Cancer Cooperative Group," Journal of Clinical Oncology, Vol. 18, No. 14, 2000, pp. 2648-2657.

[29] E. Van Cutsem, V. M. Moiseyenko, S. Tjulandin, A. Majlis, M. Constenla, C. Boni, et al., "V325 Study Group. Phase III Study of Docetaxel and Cisplatin plus Fluorouracil Compared with Cisplatin and Fluorouracil as First-Line Therapy for Advanced Gastric Cancer: A Report of the V325 Study Group," Journal of Clinical Oncology, Vol. 24, No. 31, 2006, pp. 4991-4997. doi:10.1200/JCO.2006.06.8429

[30] K. Kondo, M. Murase, Y. Kodera, S. Akiyama, K. Ito, Y. Yokoyama, et al., "Feasibility Study on Protracted Infusional 5-Fluorouracil and Consecutive Low-Dose Cisplatin for Advanced Gastric Cancer," Oncology, Vol. 53, No. 
1, 1996, pp. 64-67. doi:10.1159/000227537

[31] R. Kim, S. Murakami, Y. Ohi, H. Inoue, K. Yoshida and T. Toge, "A Phase II Trial of Low Dose Administration of 5-Fluorouracil and Cisplatin in Patients with Advanced and Recurrent Gastric Cancer," International Journal of Oncology, Vol. 15, No. 5, 1999, pp. 921-926.

[32] H. Kusumoto, Y. Maehara, T. Kusumoto, H. Anai, K. Akazawa and K. Sugimachi, "Chemosensitivity Differences between Primary and Metastatic Lesions of Linical Gastric Cancer," European Journal of Surgical Oncology, Vol. 14, No. 6, 1988, pp. 685-689.

[33] H. Miyamoto, M. Ogawa, J. Inagaki, N. Horikoshi, K. Ezaki, K. Inoue, et al., "Combination Chemotherapy of Advanced Gastric Cancer with Adriamycin, Mitomycin C, and Ftorafur," Japanese Journal of Cancer and Chemotherapy, Vol. 10, No. 4, 1983, pp. 1160-1164.

[34] Y. S. Chung, Y. Yamashita, T. Inoue, T. Matsuoka, B. Nakata, N. Onoda, et al., "Continuous Infusion of 5-Fluorouracil and Low Dose Cisplatin Infusion for the Treatment of Advanced and Recurrent Gastric Adenocarcinoma," Cancer, Vol. 80, No. 1, 1997, pp. 1-7. doi:10.1002/(SICI)1097-0142(19970701)80:1<1::AID-C NCR1>3.0.CO;2-\#

[35] J. S. Macdonald, P. S. Schein, P. V. Woolley, T. Smythe, W. Ueno, D. Hoth, et al., "5-Fluorouracil, Doxorubicin, and Mitomycin (FAM) Combination Chemotherapy for Advanced Gastric Cancer," Annals of Internal Medicine, Vol. 93, No. 4, 1980, pp. 533-536.

[36] J. Mitry, P. Taïeb, V. Artru, V. Boige, J. N. Vaillant, M. C. Clavero-Fabri, et al., "Combination of Folinic Acid,
5-Fluorouracil Bolus and Infusion, and Cisplatin (LV5FU2P Regimen) in Patients with Advanced Gastric or Gastroesophageal Junction Carcinoma," Annals of Oncology, Vol. 15, No. 5, 2004, pp. 765-769. doi:10.1093/annonc/mdh182

[37] M. Ychou, C. Astre, P. Rouanet, J. M. Fabre, B. SaintAubert, J. Domergue, et al., "A Phase II Study of 5-Fluorouracil, Leucovorin and Cisplatin (FLP) for Metastatic Gastric Cancer," European Journal of Cancer, Vol. 32A, No. 11, 1996, pp. 1933-1937. doi:10.1016/0959-8049(96)00147-5

[38] C. Louvet, F. Carrat, F. Mal, M. Mabro, K. Beerblock, J. C. Vaillant, et al., "Prognostic Factor Analysis in Advanced Gastric Cancer Patients Treated with Hydroxyurea, Leucovorin, 5-Fluorouracil, and Cisplatin (HLFP Regimen)," Cancer Investigation, Vol. 21, No. 1, 2003, pp. 1420. doi:10.1081/CNV-120016399

[39] J. Lee, T. Lim, J. E. Uhm, K. W. Park, S. H. Park, S. C Lee, et al., "Prognostic model to Predict Survival Following First-Line Chemotherapy in Patients with Metastatic Gastric Adenocarcinoma," Annals of Oncology, Vol. 18, No. 5, 2007, pp. 886-891. doi:10.1093/annonc/mdl501

[40] I. Chau, A. R. Norman, D. Cunningham, J. S. Waters, J. Oates and P. J. Ross, "Multivariate Prognostic Factor Analysis in Locally Advanced and Metastatic EsophagoGastric Cancer-Pooled Analysis from Three Multicenter, Randomized, Controlled Trials Using Individual Patient Data," Journal of Clinical Oncology, Vol. 22, 2004, pp. 2395-2403. doi:10.1200/JCO.2004.08.154

PS: performance status;

PD: pathology-poorly differentiated pathology;

PFS: progression free survival;

OS: overall survival. 